\title{
La familia centrada en el niño: impotencia y desesperanza
}

\author{
The Child-Centered Family: Impotence and Despair
}

\author{
Helena MarTín ALONSO \\ Consejería de Educación de la Comunidad de Madrid \\ helenamtn@hotmail.com \\ Marta Pizarroso FernándeZ \\ Ayuntamiento de Madrid \\ martapizarrosofernandez@hotmail.com \\ Ana SÁNCHEZ GARCÍA \\ Ayuntamiento de Madrid \\ anasanchezgarcia7@gmail.com
}

Recibido: 16/06/2013

Revisado: 03/07/2013

Aceptado: 01/10/2013

Disponible on line: 20/12/2013

\section{Resumen}

Con la propuesta de que el absentismo es un señal que habla de las dificultades en la familia, este artículo trata de explicar, a través de cuatro casos, las características relacionales familiares y entender así la función que el absentismo cumple en ellas. Para ello se analiza el marco y contextos de actuación y cómo determinan las intervenciones. Consideramos necesario ampliar la mirada para introducir posibilidades que faciliten actuaciones desde el modelo sistémico familiar, analizando los estilos parentales y la estructura familiar, y así favorecer el trabajo sobre las relaciones e interacciones de los miembros de la familia.

Palabras clave: absentismo, estructura familiar, estilos parentales, relaciones familiares.

\begin{abstract}
Working from the idea that school absenteeism is a signal that talks about difficulties in the family, this article seeks to explain, through four cases, family relational characteristics and to understand the role that absenteeism plays. We examine the framework and context of action and how they determine the interventions. We believe it is necessary to broaden a vision to introduce possibilities to facilitate performances based on the family systems model, analyzing parenting styles and family structure, thus facilitating the work on relationships and interactions of family members. Keywords: absenteeism, family structure, parenting styles, family relationships.
\end{abstract}

Referencia normalizada: Martín Alonso, H., Pizarroso Fernández, M., y Sánchez García, A. (2013): «La familia centrada en el niño: impotencia y desesperanza». Cuadernos de Trabajo Social, 26(2): 285-293.

Sumario: Introducción. 1. Una legislación, un marco de actuación. 2. Contextos de intervención: negligencia y paradoja. 3. Estudio de casos. Una intervención alternativa y esperanzadora. 4. El absentismo como una señal de malestar. 5. Conclusiones. 6. Referencias bibliográficas.

\section{Introducción}

Tanto en el imaginario social como en el abordaje actual de las investigaciones y de las propuestas de actuación, el problema del absentismo escolar se analiza tipificando a las familias y al alumnado bajo una óptica individual, lo que resulta a todas luces simplificador, como se pretende poner de manifiesto a lo largo de este artículo. Una prenoción directamente relacionada con la perspectiva simplificadora aludida es la de asociar a los menores absentistas con las familias multiproblemáticas, pertenecientes a minorías étnicas o con algún tipo de problema específico (adicción, abusos, violencia, anore- 
xia), o al estatus socioeconómico bajo. La experiencia acumulada por las tres autoras nos lleva a reflexionar sobre aquellas situaciones en las cuales las familias no responden a estos parámetros, pero el absentismo es una señal de que algo acurre en la dinámica familiar. Suponemos que el absentismo cumple una función profunda dentro del sistema familiar, que es expresión de una dificultad en la estructura y en el funcionamiento familiar.

Asimismo hay una línea de pensamiento generalizada, en parte determinada por el marco organizativo y legislativo. Plantea el absentismo escolar como la privación del derecho a la educación y el consecuente incumplimiento de un deber por los padres y las madres. En parte este planteamiento presupone que éstos o la familia, son culpables del absentismo, «no lo quieren hacer», «no se esfuerzan, «son muy permisivos», cuando es más bien la expresión de dificultades intrafamiliares, como se indicaba antes.

Las preocupaciones que subyacen a esta reflexión son las numerosas demandas (que el alumno acuda al centro en seguida, que la familia cuente el porqué de lo que está pasando, etc.) que generan dudas, inquietudes, situaciones hostiles, sensaciones de fracaso e incapacidad, y plantean preguntas que transcienden el rol meramente instrumental que las familias y las instituciones nos otorgan. Estas preguntas entre otras son: ¿qué sienten las familias? ¿cómo viven el absentismo? ¿qué función cumple en sus vidas? Estas cuestiones en sí mismas ya comportan un cambio en la mirada, la desvían hacia la familia con una perspectiva sistémica. Así se contempla el absentismo en su origen su función en la familia, y las dificultades que expresa sobre el funcionamiento familiar y la adaptación de la familia y del alumno/a a determinados contextos y momentos como, por ejemplo, en el paso de primaria a secundaria.

\section{Una legislación, un marco de actuación}

En el Programa marco de Prevención y Control del Absentismo Escolar lo define como la no asistencia regular a los centros educativos del alumnado escolarizado y comprende: el absentismo elevado, cuando las ausencias son superiores al 50 por ciento de los días lectivos; el absentismo medio, cuando se sitúan entre el 25 y el 50 por ciento; y el absentismo bajo, cuando se sitúan por debajo del 25 por ciento.
El Programa pretende establecer un proceso que facilite las respuestas globalizadas a esta problemática, garantice la continuidad y la regularización de la escolarización en el periodo obligatorio (de 6 a 16 años) en tres fases: prevención, detección e intervención.

El marco de actuación tiene dos vertientes: una coercitiva que respalda el Código penal, que en su artículo 226, donde establece:

El que dejare de cumplir los deberes legales de asistencia inherentes a la patria potestad, tutela, guarda o acogimiento familiar o de prestar la asistencia necesaria legalmente establecida para el sustento de sus descendientes, ascendientes o cónyuge, que se hallen necesitados, será castigado con la pena de prisión de tres a seis meses o multa de seis a 12 meses.

Y la otra educativa, respaldada por la defensa de los Derechos de la Infancia.

El Protocolo de absentismo se lo aplican al menor los centros educativos, que son los primeros en detectar los problemas de asistencia. $\mathrm{Si}$ el propio centro educativo no consigue un cambio en el trabajo inicial con la familia, se derivará el caso, enviándole el expediente a las Comisiones de Absentismo, situadas en las Juntas Municipales, para que las educadoras/es sociales comiencen la intervención. En este momento se cita a las familias con una carta certificada, para realizarles una entrevista formal in situ, en la Junta Municipal. En dicha entrevista los técnicos de educación les explican lo que implica el derecho y la obligatoriedad de la educación. Esta primera toma de contacto tiene un carácter coercitivo, hay una obligatoriedad para los padres, y tiene un objetivo, cambiar la actitud de los padres. No hay que olvidar que el sistema legal, en general, se sustancia en la amenaza de sanción.

Es en este momento cuando entra en juego la Sección de Educación, instancia administrativa generalmente desconocida por las familias. Es necesario realizar algunas reflexiones que trasciendan el protocolo, (el marco de actuación comporta una gran burocratización), y hacen pensar en: qué siente la familia cuando recibe la citación formal para comparecer ante los técnicos; qué expectativas tiene ante esta primera entrevista; quién es conveniente que acuda a ese primer contacto, entre otras. Según Lamas (1997), estos momentos iniciales del 
contacto de las familias con los servicios comunitarios están llenos de dificultades, pero también ofrecen innumerables oportunidades. En especial, la manera como se lleve a cabo el encuentro va a marcar la dinámica relacional entre ambos. De modo que este primer contacto con la familia del menor que se ha sido considerado absentista, se ha de guiar por la necesidad de crear un vínculo que permita acercarse y conocer la realidad de la familia.

\section{Contextos de intervención: negligencia y paradoja}

El contexto legislativo y normativo organiza en parte el de trabajo y expectativas de los protagonistas. El objetivo de la intervención está claro: la desaparición del absentismo. Sin embargo, para conseguirlo resulta determinante el análisis de las implicaciones de los contextos y metacontextos (ídem) en los cuales intervenimos.

Las familias se acercan al contexto educativo formal, esperando la prestación de unos servicios, en este caso de carácter formativo. También acuden al encuentro de un espacio en el que se va a «educar» a sus hijos, si bien en este punto pueden tener o tienen dos expectativas diferentes: entrar en confrontación con el equipo o esperar un refuerzo de su «forma de hacer» por parte de aquél. En general, transitan en un continuo de posiciones que van de aquellas en las que esperan y reciben apoyo ante la tarea parental (se refuerza y comparte su manera de hacer las cosas), a otras en las que se cuestiona e incluso se critica, implícita o explícitamente, el funcionamiento familiar. Esta dinámica genera relaciones familia-Centro que se caracterizan por el malestar e incluso por el rechazo.

La función controladora no acostumbra a estar en el «menú» de expectativas de las familias respecto a la escuela, que suelen esperar funciones de supervisión del aprendizaje y de la disciplina dentro de los centros. Pero la función de protección de los menores se ha ido introduciendo poco a poco en el contexto escolar, al tratarse de un mirador privilegiado de los chicos y chicas, lo que ha provocado mayor coordinación y trabajo conjunto con otras instituciones externas.

El contexto que defiende la Administración municipal en materia de absentismo escolar, además de ser coactivo, no deja de ser asisten- cial, adjudicando una posición de superioridad al profesional frente a la familia, lo que puede llevar, negativamente, a una desresponsabilización de los padres sobre sus propios actos y a una excesiva involucración del profesional, pareciendo que el problema del absentismo transita de los padres a los profesionales.

Es muy importante destacar cómo se genera el primer contacto entre las familias y los servicios educativos municipales, caracterizándose por la desconfianza y la ignorancia. Las familias se muestran asustadas, amenazadas y algunas enfadadas, manifestando incomprensión acerca de la citación. La mayor parte de ellas verbaliza querer que sus hijos/as acudan al colegio y no encontrar la manera de conseguirlo.

Ya en este primer contacto nace la doble función: coactiva y educativa. El hecho de tener el primer contacto punitivo con el/la técnico de educación genera en las familias un postura defensiva ante cualquier intervención. La posterior intervención de las educadoras de absentismo al plantear una entrevista cercana facilita la creación de un vínculo positivo que favorece la elaboración de posibles hipótesis, permitiendo dibujar un mapa familiar y establecer objetivos de intervención. En este momento es importante contextualizar a la familia sobre el marco de intervención y expresar los compromisos, para que, de alguna manera, se cuente con su permiso y colaboración para el cambio, lo que facilita su entendimiento e implicación.

De este modo tanto los centros como los servicios educativos municipales se sitúan como contexto de control, con las características que describe Lamas (1997), siendo la prestación del servicio obligatoria, sin que exista demanda, emitiendo un juicio, apoyado socialmente por unas normas legisladas compartidas, basadas en la protección. Uno de los problemas de este contexto es la lejanía que se genera respecto a las familias, movilizándose en ellas un sentimiento de culpa y estableciéndose una relación complementaria rígida entre los diferentes sistemas que intervienen (instituciones) y las familias.

Las intervenciones que realizan los centros escolares y las educadoras de absentismo se enmarcan así en este contexto, pero en muchas ocasiones conllevan el desarrollo de una rela- 
ción asistencial, donde la comprensión y el respeto por la decisión de la familia quedan de momento supeditadas a la propia relación, generándose dependencia y escuchándose frases del tipo: «Usted me dijo que le castigara».

Como refiere Cirillo (2005), se observa que estos contextos comparten características que van a determinar las intervenciones y las relaciones entre los diferentes sistemas como son: la ausencia de solicitud o demanda por parte de los alumnos y sus familias; la imposición del profesional; la desconfianza en él/ella; la circulación de contenidos y de información en la red; y la elaboración de un informe de resultados.

El modelo actual coactivo, punitivo y culpabilizador, implícito o explícito, que guía las actuaciones y el mensaje que se transmite a las familias, es contradictorio. Se genera un doble vínculo paradójico: por un lado, se les asiste y controla fomentando en las familias dependencia, y por otro lado se les exige autodeterminación, implicación, iniciativa y cambio. De este modo se establece una pauta circular: cuanto más se sitúa como experto el profesional y determina las actuaciones a seguir a la familia para que el absentismo disminuya, menos cambios se producen de manera espontánea y real en aquélla. Asimismo, cuanto más se aleja la familia, más coactivo se vuelve hacia ella el profesional y el contexto de protección.

El sistema, tal y como está estructurado hoy, defiende la idea de la intervención masiva de recursos externos dentro de las familias, expulsando en muchas ocasiones de dicha intervención a los propios miembros, padre, madre e hijos. Porque ¿cómo podemos entronizar a esa madre, cuando desde fuera llega «el experto» y le dice que no lo está haciendo bien? Además, el profesional lo hace en presencia del hijo, colocándola en una posición de incompetente en la crianza, lo que no contribuye a su jerarquización.

Este sistema está poblado de múltiples profesionales que no siempre acuerdan un mismo mensaje sobre qué sucede o qué decir a las familias. Esto comporta debilidad en los mensajes repetitivos incluso contradictorios; discordancias, y desencuentros; también, puede implicar alianzas, jerarquías incongruentes, $\mathrm{y}$ coaliciones entre los propios profesionales o con un miembro de la familia. En general, este tipo de actuaciones son las que favorecen las reticencias y la desconfianza de las familias en los recursos socioeducativos. En definitiva, los profesionales determinan la senda de las intervenciones, no siendo estas neutrales. En los casos de absentismo, la tipología y estilos de los profesionales son muy variados, aunque se comparte el marco de actuación determinado por la normativa.

\section{Estudio de casos. Una intervención alter- nativa y esperanzadora}

Desde la perspectiva sistémica, las primeras cuestiones que se han de plantear son: ¿qué función tiene el absentismo en cada familia?, ¿en qué contexto relacional se gesta? En general, el modelo sistémico ayuda a contextualizar este problema dentro de la familia y a comprender el papel que representa el síntoma. A continuación, a través del análisis de casos, se llevará a cabo un primer acercamiento a la existencia de ciertas circunstancias comunes que hacen patente la necesidad de realizar intervenciones con un enfoque que trascienda el pensamiento «culpabilizador» de los padres que implica el marco legislativo y organizativo.

\section{1. «Buscando a papá»}

Carlos está matriculado en un instituto público, en primaria era un alumno modelo, pero en el paso a secundaria comienzan los problemas de absentismo. En la primera entrevista familiar, a la que solo acude la madre, dice que no sabe qué hacer con su hijo, pero tampoco se muestra muy preocupada, incluso se la ve cómoda con la situación. Tras varias entrevistas, a las que nunca acude el padre, se descubre el excesivo apego que hay entre madre e hijo. Esta relación de Carlos con su madre ha generado en él un tipo de vínculo que le obliga a no querer ir al centro para no separarse de ella, y responde así a una necesidad que implícitamente demanda su madre. Carlos y su madre protagonizan una relación emocional muy intensa que posee un gran valor para ambos, los intereses de ambos se resuelven de este modo. La madre afirma que Carlos tiene que ir al Instituto, pero a la vez disculpa sus faltas de asistencia con excusas, ambas afirmaciones son contradictorias y excluyentes.

Bateson (1976) define en la Teoría del doble vínculo los efectos de dar órdenes contra- 
dictorias o paradójicas. Aunque el mensaje carece de sentido desde el punto de vista lógico, el receptor no puede dejar de reaccionar de forma apropiada, ya que el mensaje contiene en sí mismo la paradoja. Carlos repite la experiencia, de modo que percibe su universo en modelos de doble vínculo: por un lado, su madre verbaliza que tiene que ir al Instituto, pero le sigue premiando su falta de asistencia, sigue siendo el centro de atención.

La madre llega a verbalizar un día que duerme con Carlos en la habitación del niño. El niño, (ya no tan niño) ha ocupado un lugar de poder que no le corresponde. La baja jerarquización materna, que según Lamas (1997) se configura como un elemento diferenciador, permite a Carlos manipular a su madre sin que ésta sea consciente. Los límites entre madre e hijo son difusos, demasiada información pasa entre ellos, son una pareja. El estilo parental es permisivo, lo que conlleva, según Domenech, Donovich y Cowley, (2009), que existan altos niveles de sensibilidad, alta autonomía y bajos niveles de exigencia paterna. La parentalidad está representada en este caso solo por la madre, hecho que no favorece el subsistema parental frente al fraternal. El padre, a quien nunca se llegó a conocer, después de más de un año de intervención, trabaja con el hermano mayor, por lo que la casa se ha convertido en una liga de fútbol donde equipo A (madre y Carlos) y equipo B (padre y hermano mayor) no suelen compartir espacios propios de cada subsistema. Esta mezcla paterno-filial no favorece la solución de los problemas de absentismo. Carlos demanda un espacio en el Equipo $\mathrm{B}$, pero viendo que no hay cabida, se alía más con la madre.

El protocolo se cerró porque el expediente se envió a la fiscalía y la madre tenía tanto miedo de perder a su hijo que convenció a Carlos para que fuera al Instituto. Aunque el problema del absentismo ha sido solucionado, la dinámica familiar no, pero desde el Programa al cerrarse el protocolo se deja de intervenir con la familia.

\section{2. «No, sin mi hijo»}

La historia familiar ayuda a comprender las razones que empujan a los padres a actuar de una forma u otra. La madre de Sergio aprovechó el nacimiento de su hijo para refugiarse en su ma- lestar. Tras varios años de infelicidad conyugal decide separarse, el sistema conyugal está roto lo que provoca que el sistema parental se resienta. La madre dedica tal atención al niño que éste deja de acudir al colegio ( $4^{\circ}$ Primaria) a una edad muy temprana, ausencias que ignora su padre, que vive fuera ya del domicilio familiar. La madre verbaliza que sufrió malos tratos y que tiene miedo del marido, por lo que no quiere que sepa nada de las faltas de asistencia. La madre se encierra cada vez más en Sergio y justifica sus faltas de asistencia con enfermedades «ficticias».

Los límites entre madre e hijo son excesivamente difusos, el niño posee más información de la necesaria sobre la separación de sus padres. La madre se muestra cercana con su hijo, tanto que le hace partícipe de sus vivencias cotidianas. La madre le está «obligando» a elegir un bando y parece que Sergio ha caído en sus redes. El niño está jerarquizado de tal manera que su madre le entrona como el cabeza de familia, él cuida de ella y ella de él, a pesar de que hay un hermano mayor, adulto, más vinculado al padre que vive con ellos,.

Volvamos aquí a la paradoja. El niño quiere ir al colegio, cuando va se relaciona perfectamente con sus iguales y disfruta del juego y de las clases. Pero el niño siente que traiciona a su madre cuando la abandona, dejándola sola en casa. El niño vive esa encrucijada dolorosamente, pero no puede permitir que su madre se sienta herida y abandonada otra vez.

El estilo parental, aunque en este caso sería «marental» porque el padre tiene poca cabida en la vida del niño, es permisivo, existiendo altos niveles de sensibilidad, una alta autonomía y bajos niveles de exigencia materna.

Como cualquier buen héroe, Sergio se dedica a velar por su amada madre. Los roles están tan invertidos que no se sabe quién cuida y quién es cuidado. Parece así básico trabajar la desjerarquización de Sergio y la autonomía de la madre en el cuidado de su hijo. Hay que introducir la importancia de que el niño deje de llevar a cabo labores de adulto y que sea la madre quien debe velar por el bien de su hijo y no al revés. Este protocolo de absentismo sigue abierto, ya que ha sido complicado llegar al padre de Sergio. Es fundamental trabajar con ambos progenitores la relación con el niño. 


\section{3. «Encerrados en el sólo para mí»}

Luis comienza el instituto con normalidad aparente. En primaria no consta que hayan existido dificultades de absentismo y sus resultados eran buenos. Empieza a faltar con cierta regularidad para pasar a dejar de asistir durante períodos prolongados al centro. Vive con su madre en una casa, concedida por la Empresa Municipal de la Vivienda a familias con especial necesidad, y los recursos económicos provienen de una prestación social. Su padre falleció hace 5 años, siendo un tema que evitan abordar (la madre prefiere no hablar de ello y menos aún delante de Luis), no consideran que haya influido ni tenga relación con la situación actual de la familia, piensan que lo han afrontado y resuelto bien. Surge un primer gran secreto: la vida anterior a la muerte del padre y las situaciones que rodearon al fallecimiento en sí.

En las diferentes entrevistas queda patente que ni Luis ni su madre detectan un problema en las faltas de asistencia, posicionando al chico como un «enfermo», relacionándolas con temas de salud (sin aportar justificante médicos, sólo los escritos por la madre). Señalan que, cuando el chico no acude al centro, se queda en casa con su madre, indicando este hecho como algo positivo. Presume de que Luis sale poco a la calle, que tiene pocos amigos (aunque ¿hasta qué punto esto podría ser una negación de las relaciones externas del chico?), y siempre está con ella. La madre hace pocas referencias a la familia extensa, si acaso para hablar de su madre y de las cargas que supone su estado de salud actual. Y surge el segundo gran secreto: el consumo de alcohol de la madre.

La relación entre Luis y su madre es muy estrecha, parece que el hueco que ha dejado el padre lo va llenando el chico en la misión de sostener a su madre y custodiar sus secretos. Tanto es así que en una visita al domicilio la madre acaba comentando que el chico alguna vez duerme en su cama. Así, la relación entre ellos es muy fuerte, haciendo coalición respecto al exterior, quedándole a Luis pocas escapatorias, al ser casi un rehén de todas las justificaciones de la madre y de la falsa sensación de normalidad y lealtad. ¿Cómo cuestionar a una madre cuya principal dedicación es él y que le sirve de coartada en algunas situaciones?
La madre manifiesta que se siente competente para lograr que su hijo acuda al centro. Insiste en que todas sus preocupaciones por la salud de Luis demuestran «lo buena madre que es». Pero a pesar de esto, en su relación con el chico podemos detectar, siguiendo a Marrone (2001), como realiza comunicaciones culpógenas, amenazas, invalidantes de la experiencia subjetiva de Luis. Se observan claramente mensajes de doble vínculo y comentarios paradójicos, en el explícito «tienes que ir al instituto», y el implícito «estoy triste, me consuela y anima tenerte cerca, si estoy sola consumo más alcohol».

La madre muestra baja exigencia materna (ejerce poco control sobre Luis), baja autonomía otorgada (no permite la expresión individual ni la autonomía del chico) y baja calidez (no llega a implicarse y mostrar interés por las actividades que realiza Luis, le escucha y le apoya poco) pudiendo clasificarse su estilo parental primario como negligente (Domenech, Donovich y Crowley, 2009).

El absentismo moviliza a esta familia en la medida que recibe una presión coactiva de los Servicios Sociales, mostrándose la madre resistente a que se «entre» en su familia y aparentemente sorprendida por la preocupación y alarma de las profesionales. En cierto momento éstas fueron viendo como la situación no cambiaba en el sentido de su alerta, que iban descubriendo nuevas cosas que les preocupaban y que sus herramientas para acceder a la familia no funcionaban, movilizándose sentimientos de impotencia y enfado con la madre por su manera de «cerrar puertas».

Así, ante las faltas de huecos para acceder, se plantea la posible suspensión de la prestación económica. Y de esta manera la madre se acopla a las demandas de las profesionales, entrando en relación de complementariedad con ellas, admitiendo la mayor parte de las dificultades señaladas y dejando que entrara en su familia una educadora.

Tras el análisis surgen las preguntas ¿es un éxito que la madre se sienta incompetente?, ¿es un avance que delegue sus funciones en una profesional? Pero estas dudas se solventaron rápido, porque esta madre tiene múltiples habilidades y «lucha» por su funcionamiento familiar, así que al poco tiempo Luis comenzaba a asistir con más regularidad al centro, devol- 
viéndonos un mensaje claro: idos de mi familia, lo que os preocupaba ya está arreglado, ya no necesitamos nada de vosotras. Y, curiosamente, el chico en el Instituto comienza a presumir de sus broncas con la madre...

\section{4. «Un divorcio difícil»}

Los padres de Sergio y Cristian se separaron hace dos años. Ahora viven con su madre, y los fines de semana alternos los pasan con su padre. Tras la separación la madre comenzó una relación sentimental con un hombre que se trasladó al domicilio familiar. Esta persona recurría al alcohol asiduamente, hábito que al parecer comenzó a adquirir también la madre. $\mathrm{Al}$ cabo del tiempo la relación se rompió. Cristian, Sergio y su madre no hablan de este episodio. Cristian en este momento comienza a tener conductas disruptivas en el Instituto, entrando en escalada simétrica con los profesores y buscando la expulsión. Finalmente decide que no quiere seguir asistiendo, y se encierra en su domicilio. Desde el centro educativo, tras intervenir con él y sus padres para que vuelva a asistir a clase, proceden a abrir el expediente de absentismo enviándolo al Ayuntamiento. A Sergio se le abre un protocolo dos meses después del que se ha abierto a su hermano mayor, que copia y repite su conducta.

Desde la Junta Municipal se cita a los padres para la primera entrevista. La madre se muestra preocupada y manifiesta que no sabe qué hacer para convencer a Cristian, y al mismo tiempo que justifica su absentismo, alegando que el centro educativo no toma las medidas adecuadas, se coaliga con Cristian frente al centro, y lanza sobre él dobles mensajes contradictorios: por un lado demanda implícitamente sus cuidados, pero explícitamente cuando la Administración le presiona se muestra coactiva con sanciones que nunca cumple. En la entrevista se observa la preocupación y la colaboración del padre, hecho que irrita a la madre.

En sucesivas entrevistas se observa que los límites y las fronteras entre los miembros están difusos, Cristian y Sergio disponen de mucha información y están involucrados en los asuntos y conflictos conyugales. Parece haber mucho aglutinamiento entre los miembros, todos saben de todos. Tal y como refiere Díaz-Usan- divaras, (1986) con la ruptura del matrimonio se ha perdido la coparentalidad, las jerarquías no están bien definidas y las normas no son claras. Cristian decide qué, cuándo y cómo, ejerciendo la función de "hijo crecido», y reproduce este comportamiento en el aula, desarrollando estrategias de dominación con el resto de iguales y de adultos. Cuando sucede esto, el centro le expulsa.

En una de las entrevistas se percibe el estilo permisivo y negligente de su madre. Existen altos niveles de sensibilidad, excesiva autonomía otorgada y bajos niveles de exigencia paterna. La madre reconoce que les deja jugar a la play día y noche por miedo a que «la líen fuera», pero lo que en realidad se detecta es su preocupación por que se puedan enfadar con ella y perder ese privilegio de alianza que ha obtenido frente al padre.

Durante las sucesivas intervenciones se sospecha que existe un exceso de vinculación entre Cristian y su madre, que aparentemente mantienen un apego ansioso ambivalente, la separación entre ellos les genera ansiedad. La madre muestra los límites débiles y la inseguridad en la autoridad hacia su hijo mayor. Existe poco control. Las necesidades de la madre no están resueltas, por lo que prioriza las suyas y no las de su hijo. Cristian se pone celoso por las relaciones de su madre con el exterior y viceversa, de tal manera que cuando no asiste a clase y su madre vuelve del trabajo, ambos están tranquilos. El sistema se cierra cada vez más y el contacto con el exterior (centro educativo) supone una intromisión entre ambos. Cada vez que el centro expulsa a Cristian la relación entre su madre y él se blinda más.

En un principio la relación entre los hermanos es débil y problemática, dándose incluso comportamientos agresivos. Sin embargo cuando aparece el absentismo también en Sergio, a ambos se les identifica como absentistas y como «el problema», por lo que la rabia y la supervivencia favorecen y fortalecen la unión entre ambos frente al sistema familiar y educativo.

¿Qué función cumple el absentismo en esta familia? ¿Qué implica en las relaciones entre los miembros? ¿Cómo repercute en la ex-pareja? El absentismo, por un lado, sirve como excusa para desviar el conflicto de las relaciones intrafamiliares, triangulando con el centro edu- 
cativo; y por otro lado, permite que entren dentro de las relaciones familiares otros agentes y sujetos externos, favoreciendo que el sistema cerrado que están manteniendo los miembros de la familia vaya abriéndose poco a poco. También, se empodera y fortalece la fratría. Los hermanos se unen y, a través de sus faltas de asistencia, reclaman que los padres de algún modo se encuentren y comiencen a plantearse la coparentalidad, implicando al padre que se mostraba periférico en un principio y responsabilizando a la madre.

Actualmente el expediente de Cristian, después de haber comenzado a asistir a un aula de compensación educativa, se ha cerrado por haber cumplido 16 años. Sergio asiste al mismo Instituto con más regularidad debido a la presión ejercida por las diferentes entidades de la Administración y haber sido derivada la familia al Centro de Atención a la Infancia (CAI). Sus faltas siguen siendo muy elevadas y cuando asiste su comportamiento es disruptivo, por lo que permanece largas temporadas expulsado.

\section{El absentismo como una señal de malestar}

Tal como se ha ido viendo a lo largo del artículo, las familias con miembros absentistas presentan dinámicas disfuncionales. Cuando se realiza un análisis de la estructura, (Minuchin, 1977), los casos planteados reflejan familias que se conforman como sistemas cerrados, rígidos, con límites muy difusos, con dificultades en el proceso de diferenciación y jerarquías confusas. En estas estructuras el padre queda fuera y la relación madre-hijo es muy intensa, porque se ha establecido a través de un apego ansioso-ambivalente que, siguiendo la explicación de Bowlby (1998), se produce cuando el cuidador está física y emocionalmente disponible sólo en ciertas ocasiones y posee unas habilidades sociales inconsistentes, lo que provoca en el niño una angustia intensa y una mezcla de comportamientos de apego con expresiones de protesta, enojo y resistencia.

Este tipo de vínculo madre-hijo se fortalece en la medida en que aquélla oculta al padre o minimiza el tema de las faltas del colegio, quedándose con el hijo y la gestión de las cosas que le incumben como única responsable de los asuntos escolares. Después de una historia de cercanía y complicidad entre madre-hijo a lo largo de la infancia, en la etapa de la adolescen- cia la madre se mueve entre posiciones casi antagónicas: por un lado, los sentimientos que genera ver que su hijo no se adapta a un sistema «normalizado» (la escuela) y por el otro, aquellos que surgen cuando ve que su hijo se queda cerca de ella. Y aparece en esta familia una situación dilemática: ¿cómo castigar, enfrentar o poner normas a los chicos justo ahora, cuando por el momento evolutivo en que se encuentran se alejan de manera natural de las familias? Esto podría poner en juego la cercanía y la falsa sensación de normalidad familiar. Por otro lado, el padre sigue aislado y periférico, recluido en la vida laboral, cada vez más apartado de las cuestiones que están afectando a su hijo, y encontrándose la situación desbordada cuando actúan los servicios de control.

Estas familias ante la imposibilidad de introducir cambios e información del exterior generan un síntoma, el absentismo, que regula las relaciones intrafamiliares y permite mantener el equilibrio interno frente al entorno. El absentismo funciona como eje visibilizador, como catalejo de esta disfunción, es el efecto llamada. Las familias ponen en marcha dicho mecanismo de manera inconsciente para alertar de que algo les está pasando. Se puede decir que es el espejo que anuncia la existencia de una problemática y que alerta a los diferentes recursos para que la aborden. Pero una vez activada «la alarma» es prioritario analizar para qué está sirviendo el absentismo en la familia, no quedándose simplemente en el porqué, pasando a encontrar la demanda real del alumno y de su familia, y abordando el absentismo como una señal de malestar que afecta a todos los miembros y que se tiñe de diferentes significados para cada uno ellos.

Es necesario, pues, luchar contra la concepción lineal e individual prominente, a lo que ayudará la incorporación de todos los componentes de la familia, para poder desviar el síntoma (el absentismo) y no perpetuar en los recursos la idea de la madre en casa y el padre en el mundo laboral. Asimismo es necesario trabajar sobre la responsabilización de los miembros en la dinámica familiar y conseguir cambios, trayendo narraciones distintas de las que expone la familia, rescatando de su historia familiar las capacidades y momentos positivos de los miembros y de la familia. Además, teniendo en cuenta que la familia es un sistema 
en interrelación, se deben ahondar y valorar las relaciones y las interacciones entre los miembros de la familia y de estos con el resto de los contextos y sistemas (Escuela, Servicios Sociales, Administración, etc.).

La importancia del contexto educativo se torna crucial, ya que en el momento actual los centros cada vez se están constituyendo más como sistemas cerrados y muy rígidos y se está provocando que entre miembros básicos de la comunidad educativa (familia y profesorado) circule poca información y cada vez de una manera más burocratizada y culpabilizadora, en la que ambos sistemas (familia y escuela) comparten sentimientos ambivalentes de impotencia, desesperanza y de heteroresponsabilización. Con pocos recursos para prevenir, detectar e intervenir, con tutores y tutoras sobrecargados, profesionales a media jornada, sin becas ni profesorado especializado, con aulas sobrecargadas, con una valoración cada vez mayor del malentendido esfuerzo individual del alumnado (que mide indirectamente el tergiversado esfuerzo de sus familias) ¿Es esta escuela la que camina hacia la disminución del absentismo?

\section{Conclusiones}

Cuando el cambio se centra únicamente en el alumno que debe asistir al centro obligatoria- mente y se señala principalmente a la familia como responsable de que el «se obre milagro», la misión se convierte en casi imposible, generando sentimientos de impotencia y desesperación en todas las partes implicadas (familias, alumnos, centros educativos y educadoras de absentismo).

La escasez de recursos y la mala gestión no ayudan a comprender la complejidad de las situaciones familiares. El trabajo con las familias conlleva un tiempo que hoy en día es escaso $\mathrm{y}$ en algunos momentos inexistente. ¿Se pretende buscar una solución del problema o solo que desaparezca lo que se ve, el absentismo)? ¿Se generan así contextos de colaboración y comprensión por parte de todos o relaciones en las que las profesionales saben cuál es la solución genérica, hablan y hablan sin curiosidad y se posicionan en el poder? La intervención familiar conlleva, en muchas ocasiones, un esfuerzo mental y emocional fuerte. Trabajar con personas genera relaciones que siempre comportan cambios personales, que se producen no solo en los usuarios, sino también en el educador. Por ello, otro de los elementos relevantes es la presencia de una segunda mirada, que bien podría surgir del trabajo en red o dentro del propio programa de absentismo, con la intervención de dos educadoras con cada familia.

\section{Referencias bibliográficas}

Bateson, G. (1976). Pasos hacia una ecología de la mente. Buenos Aires: Lohle.

Boletín Oficial del Estado. (1995). Ley Orgánica 10/1995, de 23 de Noviembre del Código Penal. BOE, 281. Madrid: BOE.

Bowlby, J. (1998). El apego y la pérdida. Barcelona: Paidós.

Cirillo, S. (2005). Malos padres. Barcelona: Gedisa.

Comunidad de Madrid y Ayuntamiento de Madrid. (2001). Convenio de colaboración para la prevención y el Control del Absentismo Escolar. Madrid.

Diaz-Usandivaras, C. (1986). Ciclo del divorcio en la vida familiar. Revista de Terapia familiar, 15. Buenos Aires.

Domenech Rodríguez, M., Donovich, M. y Crowley, S. (2009). Estilos parentales en un contexto cultural, observaciones del «estilo protector» en latinos de primera generación. Family Process, 48 (2). Recuperado de: http://www3.intersciencie.wiley.com/journal/117959054/home.

Lamas, C. (2007). Para comprender la adolescencia problemática. Revista Redes, 63-85.

Lamas, C. (1997). Los primeros contactos. En L. Coletti y J. L. Linares (comps.), La intervención sistémica en los servicios sociales ante la familia multiproblemática. Barcelona: Paidós.

Marrone, M. (2001). Las disfunciones de la parentalidad. En VV.AA. La teoría del apego. Un enfoque actual. Madrid: Psimática

Minuchin, S. (1977). Familias y terapia familiar. Barcelona: Gedisa. 